\title{
Cryopumping of atomic hydrogen
}

\author{
V. G. Luppov and M. Mertig ${ }^{\text {a) }}$ \\ Laboratory of High Energies, Joint Institute for Nuclear Research, P.O. Box 79, Head Post Office, \\ Moscow, USSR \\ T. Roser, ${ }^{\text {b) }}$ B. S. van Guilder, and B. Vuaridel ${ }^{\text {c) }}$ \\ Randall Laboratory of Physics, The University of Michigan, Ann Arbor, Michigan 48109-1120 \\ Yu. M. Melnik and A. F. Prudkoglyad \\ Institute for High Energy Physics, 142284 Protvino, Moscow Region, USSR
}

(Received 18 April 1991; accepted for publication 28 July 1991)

The pumping speed for the cryopumping of an atomic hydrogen beam was measured.

Measurements were made for cryocondensation, cryosorption, and differential pumping. The pumping speed for atomic hydrogen was observed to be much smaller than the pumping speed for molecular hydrogen. It is believed that this is due to the energy released during the recombination of the atomic hydrogen.

\section{INTRODUCTION}

A high-density ultracold spin polarized atomic hydrogen jet ${ }^{1}$ will be used as an internal target for the experiments NEPTUN and NEPTUN-A, ${ }^{2}$ which will study spin effects in proton-proton scattering at energies of $400 \mathrm{GeV}$ and $3 \mathrm{TeV}$ at the UNK accelerator being built at Protvino, USSR. The ultimate intensity goal of this jet is a polarized beam of $10^{18}$ atoms $/ \mathrm{s}$. In order to keep the vacuum pressure inside of the accelerator chamber at about $10^{-9}$ Torr, it is necessary to capture the vertical jet after the interaction with the accelerator beam in a catcher with a pumping speed of $\sim 3 \times 10^{7} \mathrm{f} / \mathrm{s}$. If the catcher does not take advantage of differential pumping and has a cryopanel surface area of $12 \mathrm{~m}^{2}$, then a pumping speed of $8.8 \times 10^{21}$ atoms/(Torr $\mathrm{s} \mathrm{cm}^{2}$ ) will be necessary.

Here we present the first experimental results of cryopumping of atomic hydrogen. The measurements were performed in a prototype catcher where we guided the flow of gas onto the cryosurfaces. This simulated the situation in the NEPTUN experiment where the atomic beam will be directed into a catcher after interaction with the UNK beam. In this case only atoms that are not trapped can escape back to the accelerator chamber. Under these conditions the pumping speed is not expected to be limited by the thermal velocity of the gas particles.

\section{EXPERIMENTAL APPARATUS AND PROCEDURE}

The prototype catcher (Fig. 1) was designed to measure the pumping speed by means of a constant pressure method. For this purpose the gas is fed into the catcher with a constant flow $\phi$ and the equilibrium pressure $P_{\mathrm{RT}}$ is measured at room temperature. The pumping speed $S$ can be calculated from the expression

\footnotetext{
") Permanent address: Sektion Physik der Technischen Universität Dresden, Mommsenstr. 13, 8027 Dresden, Germany.

${ }^{6}$ Permanent address: AGS Department, Brookhaven National Laboratory, Upton, NY 11973.

'Permanent address: Départment de Physique Nucléaire et Corpusculaire, Université de Genève, $\mathrm{CH}-1211$ Genève 4 , Switzerland.
}

$$
S=\phi /\left(P_{\mathrm{RT}}-P_{\mathrm{RT}}^{\text {back }}\right),
$$

where $P_{\mathrm{RT}}^{\text {back }}$ is the measured background pressure under the same experimental conditions for a flux $\phi=0$.

The catcher is installed in a ${ }^{4} \mathrm{He}$ bath, which can be pumped to about 100 Torr to lower the temperature to 2.7 $K$. The catcher consists of a brass pot with a copper bottom flange on which different types of cryopanels can be mounted. The tube connecting the brass pot to the roomtemperature flange included a vacuum jacket in order to keep the active pumping surfaces at a constant temperature independent of the helium level. To imitate the NEPTUN experiment configuration, hydrogen atoms produced in a high-flux $\mathrm{rf}$ dissociator are directly transported to the bottom of the catcher through a Tefion tube that is $120 \mathrm{~cm}$ long and $1.25 \mathrm{~cm}$ in diameter. The flow of molecular hydrogen fed into the dissociator is controlled by a Hastings Mass Flow Meter CPR-4A which has an accuracy of $\pm 2 \%$.

The atomic hydrogen flow into the catcher was determined by measuring the change of the evaporation rate from the helium bath due to the energy released during the recombination of hydrogen atoms at the cryogenic surfaces. This allowed us to determine the dissociation efficiency after the transport of the atoms through the Teflon tube. The temperature of the Teflon tube was kept above 80 $\mathrm{K}$. We have measured an efficiency of $\sim 60 \%$ and $\sim 30 \%$ for a feedrate of 1 and $4 \mathrm{sccm} / \mathrm{min}$, respectively of molecular hydrogen into the dissociator.

The pressure in the catcher was measured with a cold cathode gauge, Balzers IKR-020, whose accuracy is approximately $\pm 5 \%$ during short periods of operation and between $+60 \%$ and $-50 \%$ of an absolute mean for longterm operation. The gauge was installed at the top of a 90-cm-long and 1.65-cm-i.d. stainless-steel tube, the bottom end of which was located over the cryopanels. The measured room-temperature pressure was corrected for the reduced ionization efficiency of hydrogen.

The background pressure, $p^{\text {back }}$ was determined by covering the cryopanel surface $\left(818 \mathrm{~cm}^{2}\right)$ of the closed catcher volume with a $\mathrm{H}_{2}$ surface coverage of $\sim 1 \times 10^{17}$ 


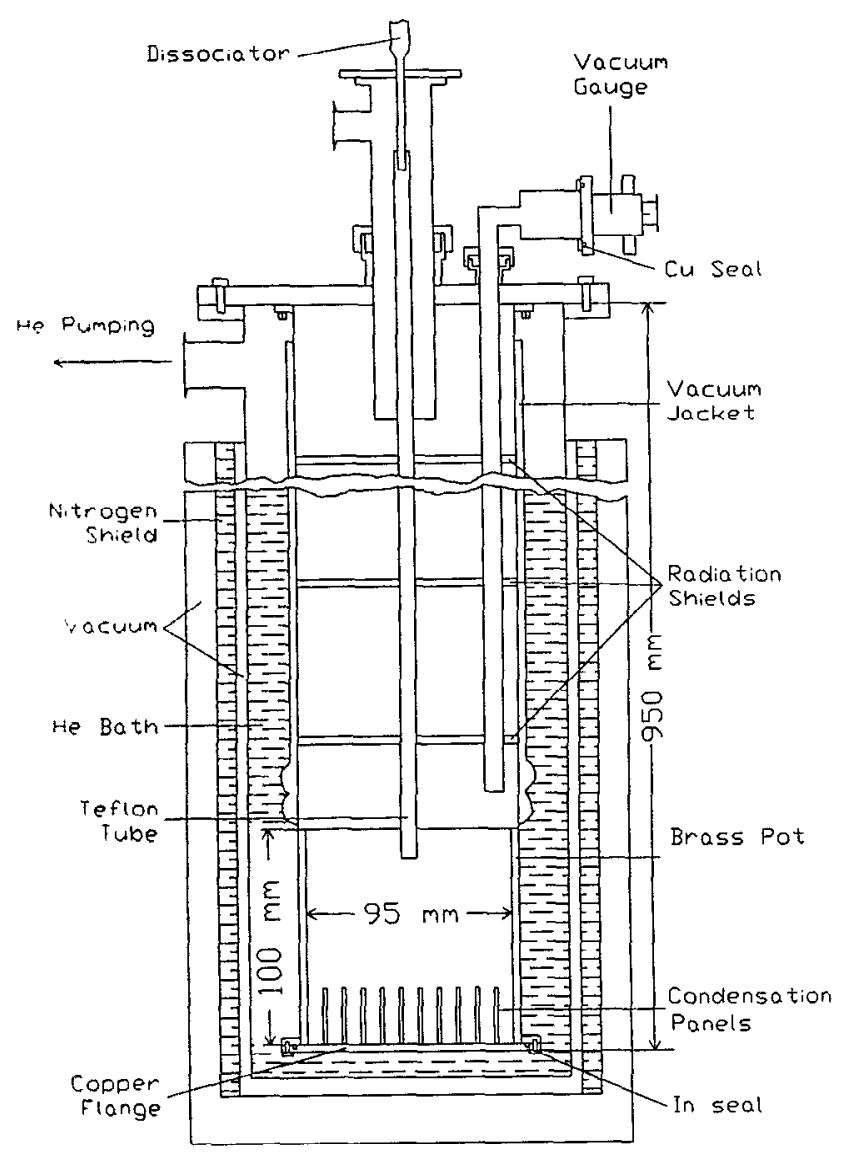

FIG. 1. Design of the prolotype catcher.

$\mathrm{mol} / \mathrm{cm}^{2}$ and measuring the temperature dependence of the pressure. The results are shown in Fig. 2, where data from the literature are also presented. For temperatures above $4 \mathrm{~K}$, the measured pressure follows the equilibrium

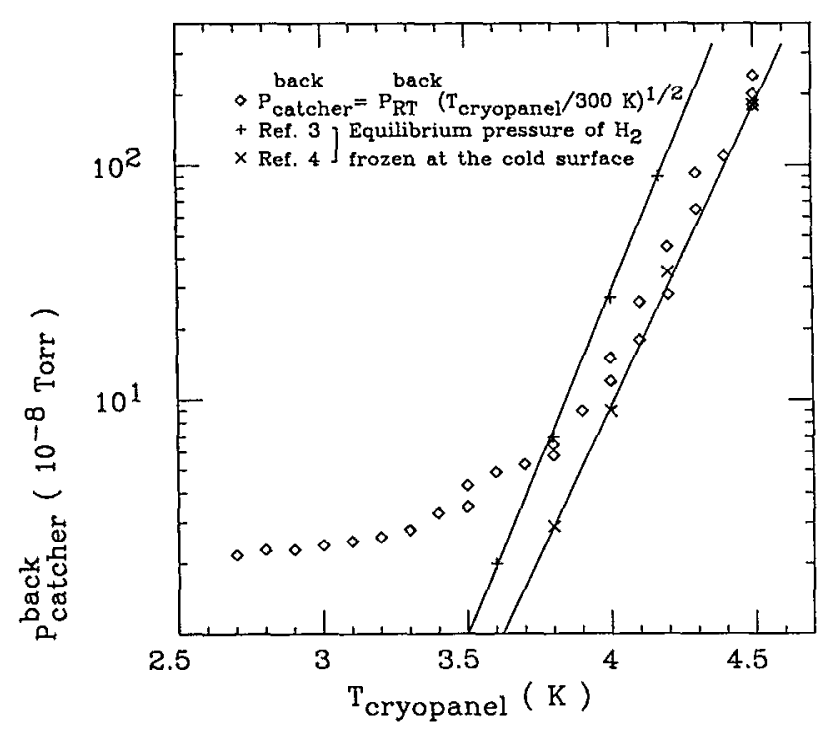

FIG. 2. Background pressure in the catcher with frozen $\mathrm{H}_{2}$ coverage as a function of cryopanel temperature.

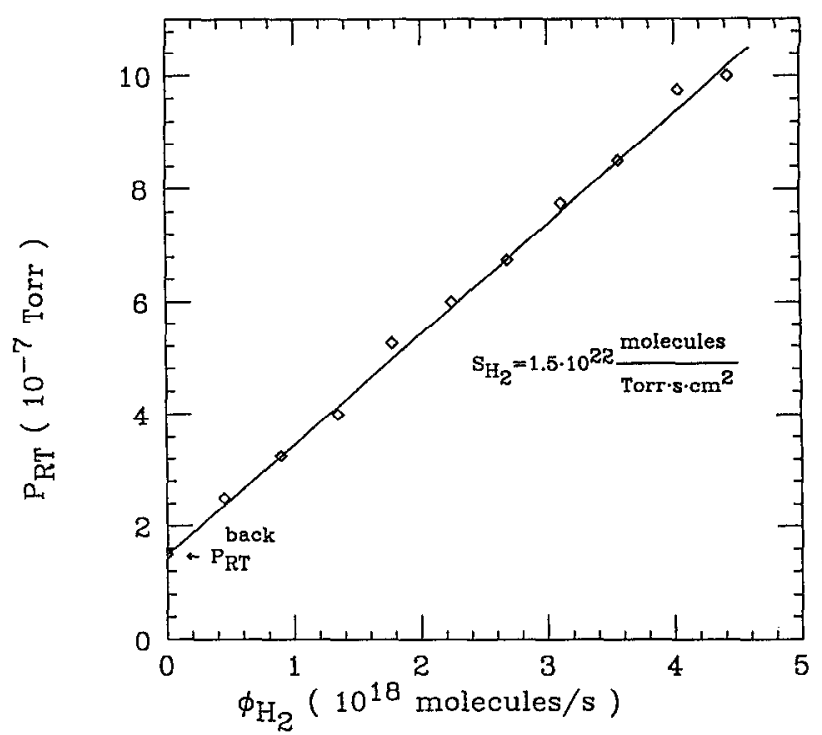

FIG. 3. Equilibrium room-temperature pressure as a function of the molecular hydrogen flux.

vapor pressure of solid hydrogen, but for lower temperatures, a saturation was observed at a level of $2 \times 10^{-8}$ Torr. This temperature-independent part of the background pressure was due to outgassing processes in the long tube connected to the pressure gauge.

In order to decrease this lower pressure limit for the experiments with charcoal and differential pumping, the pressure gauge tube was replaced by a double-walled ( 1.65 and $1.0 \mathrm{~cm}$ i.d.) stainless-steel tube with a heater and insulation wound around the outside of the inner tube. After baking out the tube at $120^{\circ} \mathrm{C}$ for a few days, a minimum pressure of $4 \times 10^{-9}$ Torr was achieved. In all measurements the background pressure was less than $15 \%$ of the measured equilibrium pressure.

\section{RESULTS}

\section{A. Cryocondensation pumping}

In order to compare the pumping speed of atomic hydrogen with that of molecular hydrogen, we first measured the pumping speed of molecular hydrogen at $3.0 \mathrm{~K}$ for a cryopanel area of $340 \mathrm{~cm}^{2}$. Figure 3 shows the equilibrium pressure in the catcher as a function of the flux of hydrogen molecules into the catcher. From these results a pumping speed of $S_{\mathrm{H}_{2}}=1.5 \times 10^{22} \mathrm{~mol} /\left(\right.$ Torr s $\mathrm{cm}^{2}$ ) can be calculated, which corresponds to $430 \ell /\left(\mathrm{s} \mathrm{cm}^{2}\right)$. This value is approximately a factor of 10 grcater than the maximum values reported in the literature [for instance, $S_{\mathrm{H}_{2}}=38$ $\left.\ell /(\mathrm{s} \mathrm{cm})^{2}\right]$. These values are close to the upper limit of $\sim 45 \ell /\left(\mathrm{s} \mathrm{cm}^{2}\right)$ for pumping a vessel with room-temperature hydrogen. We believe that the difference between our measured value and the values reported in the literature is caused by the special geometry of our experiment as discussed.

In Fig. 4 experimental results for atomic hydrogen are shown for two different cryopancl arcas at $3.0 \mathrm{~K}$. A com- 


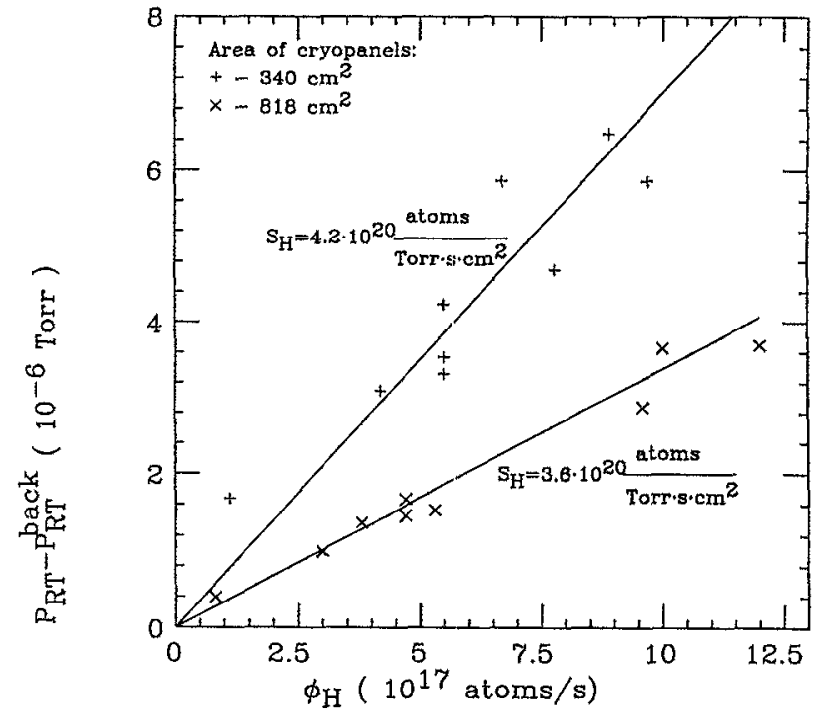

FIG. 4. Equilibrium room-temperature pressure corrected for the background pressure as a function of the atomic hydrogen flux.

parison of the results for atomic and molecular hydrogen under the same experimental conditions $\left(A_{\text {cryopanel }}=340\right.$ $\mathrm{cm}^{2}$ ) shows that the pumping speed for atomic hydrogen $S_{\mathrm{H}}=4.2 \times 10^{20}$ atoms/(Torr s cm$\left.)^{2}\right)\left[\sim 12 \ell /\left(\mathrm{s} \mathrm{cm}^{2}\right)\right]$ is approximately a factor of 36 less than for molecular hydrogen. The observed decrease of the pumping speed is probably related to the fact that the atoms recombine at the cryosurface and increase the recombination energy of $4.48 \mathrm{eV}$ per molecule, which corresponds to a temperature of $\sim 52000 \mathrm{~K}$. The high-energy molecules have to be thermalized by multiple collisions with the surface before sticking. This process would lead to a decrease of the average sticking coefficient of the molecules and consequently to a decrease of the pumping speed.

Figure 4 also shows that an increase of the cryopanel surface area leads to a decrease of the measured equilibrium pressure. However, the measured pumping speed per unit surface area decreased with increasing surface area as is shown in Table I. This was probably caused by a nonuniform distribution of the incident hydrogen atoms over the cold surface. Another contributing factor may have been the shape of the cryopanels and their geometric relationship to the beam. The two largest surface areas were formed by attaching a copper plate that had been rolled into a spiral to the bottom flange. Thus, all of the surfaces

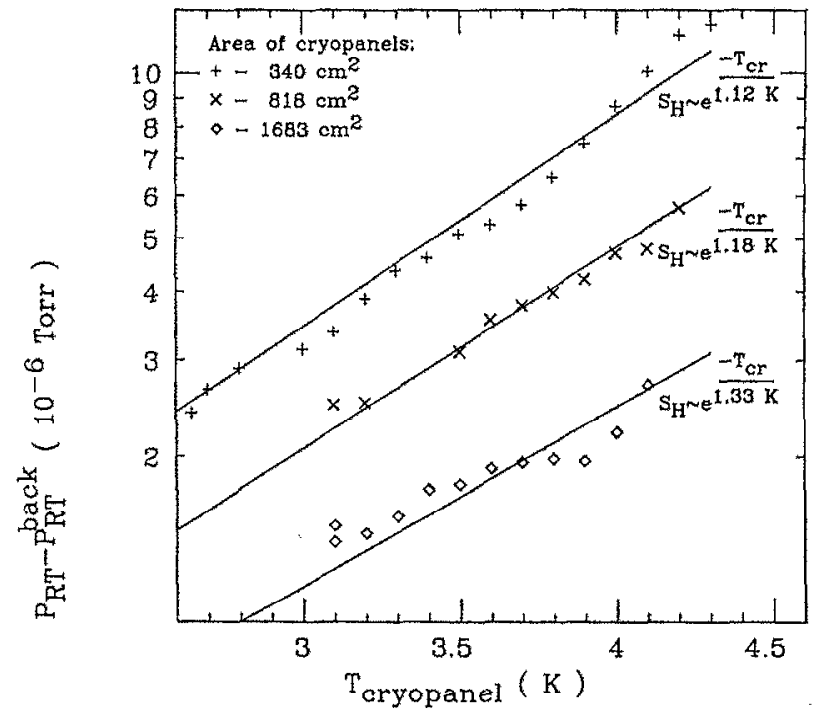

FIG. 5. Equilibrium room-temperature pressure corrected for the back: ground pressure as a function of the cryopanel temperature.

facing away from the center were not directly struck by the beam but only by particles that had already had at least one collision with the cryosurface.

For each surface area which we investigated, we obtained exponential dependence of the equilibrium pressure on the temperature of the cryosurface. The pressures measured for a flux of $\phi_{\mathrm{H}} \sim 4 \times 10^{17}$ atoms/s are shown in Fig. 5 as a function of the cryopanel temperature. From these results an increase of the pumping speed for atomic hydrogen by a factor of $\sim 2$ can be expected from lowering the temperature of the cryopanels to the lambda point of ${ }^{4} \mathrm{He}$.

\section{B. Cryosorption pumping}

Measurements of the pumping speed for cryosorption of molecular and atomic hydrogen on activated charcoal were made at a temperature of $4.2 \mathrm{~K}$. The charcoal was bonded with stycast 2850 GT epoxy to a cylindrical copper surface which was mounted on the catcher bottom flange. The total cryosorption pumping area was approximately $360 \mathrm{~cm}^{2}$. Before each measurement the charcoal was activated by baking under vacuum at about $90^{\circ} \mathrm{C}$ for approximately $5 \mathrm{~h}$. The pumping speed measured for molecular hydrogen was $S_{\mathrm{H}_{2}}=2.2 \times 10^{21} \mathrm{~mol} /\left(\right.$ Torr $\left.\mathrm{s} \mathrm{cm}^{2}\right)[63$

TABLE I. Pumping speed of molecular and atomic hydrogen for different types of cryopumps.

\begin{tabular}{|c|c|c|c|c|}
\hline & $\begin{array}{c}\text { Area of } \\
\text { cryopanel }\end{array}$ & $T(\mathrm{~K})$ & $S_{\mathrm{H}_{2}}\left[\mathrm{~mol} /\left(\right.\right.$ Torr s cm $\left.\left.\mathrm{cm}^{2}\right)\right]$ & $S_{\mathrm{H}}\left[\right.$ atoms $\left.\left./(\text { Torr s cm})^{2}\right)\right]$ \\
\hline \multirow[t]{2}{*}{ Crycondensation } & $340 \mathrm{~cm}^{2}$ & 3.0 & $1.5 \times 10^{22}$ & $4.2 \times 10^{20}$ \\
\hline & $1683 \mathrm{~cm}^{2}$ & 3.0 & & $1.9 \times 10^{20}$ \\
\hline Cryosorption & $360 \mathrm{~cm}^{2}$ & 4.2 & $2.2 \times 10^{21}$ & $8.8 \times 10^{20}$ \\
\hline Differential pumping & $1210 \mathrm{~cm}^{2}$ & 3.0 & & $6.7 \times 10^{21}$ \\
\hline
\end{tabular}




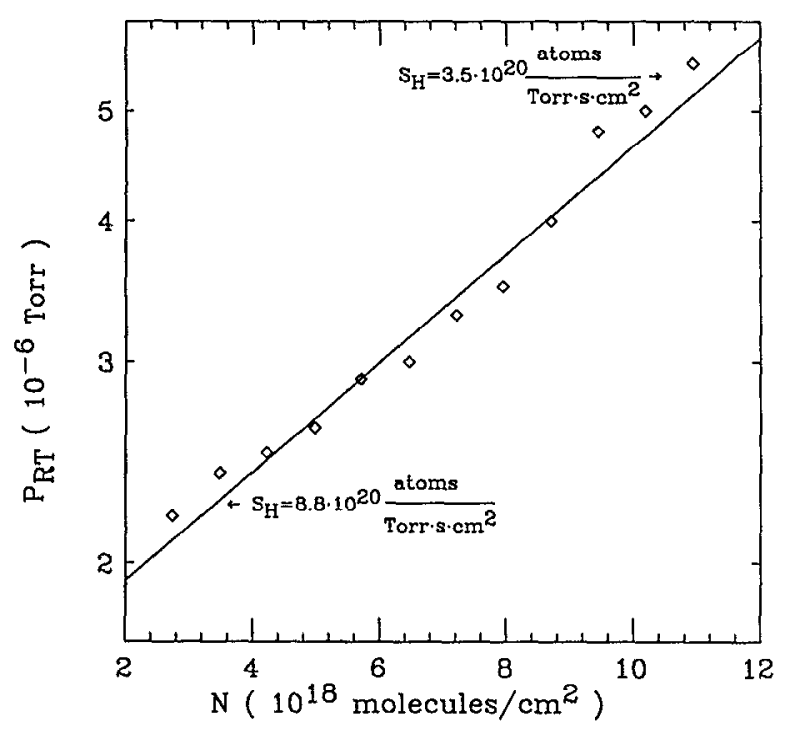

FIG. 6. Equilibrium room-temperature pressure as a function of the molecular hydrogen surface coverage of charcoal.

$\ell /\left(\mathrm{s} \mathrm{cm}^{2}\right)$ ], which is less than the pumping speed for cryocondensation of molecular hydrogen $\left[1.5 \times 10^{22}\right.$ $\mathrm{mol} /\left(\right.$ Torr $\left.\left.\mathrm{s} \mathrm{cm}^{2}\right)\right]$. Measurements with atomic hydrogen gave a pumping speed $S_{\mathrm{H}}=8.8 \times 10^{20}$ atoms/(Torr s cm$)^{2}$ ) $\left[25 \ell /\left(\mathrm{s} \mathrm{cm}^{2}\right)\right]$, but this value quickly decreased with increasing surface coverage. The rise of the measured pressure with increasing surface coverage for an atomic hydrogen flow of $6 \times 10^{17}$ atoms/s is shown in Fig. 6. No corresponding decrease of the pumping speed was observed for cryocondensation pumping. The pumping speed, $S_{\mathrm{H}}=4.2 \times 10^{20}$ atoms $/(\text { Torr s cm})^{2}$ ) for the 340 $\mathrm{cm}^{2}$ cryocondensation panel remained constant with an atomic hydrogen flow of $6 \times 10^{17}$ atoms/s and a surface coverage of up to $40 \times 10^{18} \mathrm{~mol} / \mathrm{cm}^{2}$.

\section{Differential pumping}

In order to achievc a further increase of the pumping speed, we tested a differential pumping configuration (see Fig. 7) by dividing the catcher volume into two volumes. In the inner volume, with a small entrance hole, atoms recombine and the molecules are partially thermalized and pumped. The residual gas which leaves the inner volume is then pumped by the outer cryosurfaces. This special type of differential pumping can only be used in a beam catcher because the atomic hydrogen beam can be directed to the bottom of the catcher.

The area of the inner volume and total area of the cryosurfaces were 690 and $1210 \mathrm{~cm}^{2}$, respectively. The test configuration was designed to resemble the conditions of the NEPTUN experiment by placing the bottom end of the atomic hydrogen Teflon transport tube inside of the inner volume. The openings for the flows into and out of the inner volume both had an area of $1.2 \mathrm{~cm}^{2}$.

Measurements were made at a temperature of $3 \mathrm{~K}$ and an atomic hydrogen flux of up to $6.5 \times 10^{17}$ atoms $/ \mathrm{s}$. The

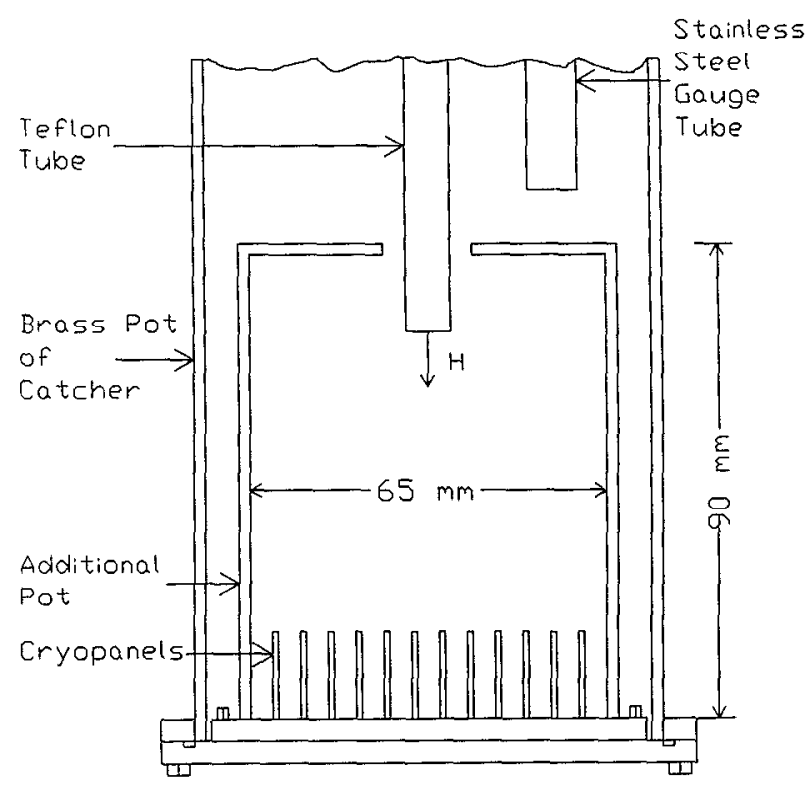

FIG. 7. Design of the bottom part of the cateher for differential pumping.

effective pumping speed (for the total cryopanel area) of atomic hydrogen for this geometry was $6.7 \times 10^{21}$ atoms/(Torr s cm$\left.{ }^{2}\right)\left[190 \ell /\left(\mathrm{s} \mathrm{cm}^{2}\right)\right]$, which is a factor of 16 larger than the pumping speed for cryocondensation pumping without differential pumping.

Using the results of our measurements for cryocondensation pumping, the gas flow out of the inner volume and the pressure outside of the pot can be estimated. If we then assume that only thermalized molecules escape from the inner volume, we can use the cryocondensation pumping speed for nondirected $\mathrm{H}_{2}$ [40 $\left.\mathrm{\ell} /\left(\mathrm{s} \mathrm{cm}^{2}\right)\right]$ to estimate the effective pumping speed for this differential pumping configuration. This simple model gives a pumping speed that is a factor of 7 larger than the measured value and therefore does not describe the situation adequately.

\section{ACKNOWLEDGMENT}

This work was supported by the U.S. Department of Energy.

${ }^{1}$ T. Roser, D. G. Crabb, W. A. Kaufman, R. S. Raymond, J. A. Stewart, B. Vuaridel, and G. R. Court, Nucl. Instrum. Methods A 301, 42 (1991).

${ }^{2}$ A. D. Krisch, "Status of NEPTUN-A Experiment," in Proceedings of the Workshop "Physics at UNK," edited by N. Tyurin (IHEP, Protvino, USSR), p. 152; V. L. Solovianov, ibid.

${ }^{3}$ C. Benvenuti, R. S. Calder, and G. Passardi, J. Vac. Sci. Technol. 13, 1172 (1976).

${ }^{4}$ M. P. Malkov, Handbook of Low Temperature Physics (Russian Edition) (Publishinghouse Energy, Moscow, 1985), p. 104.

${ }^{5}$ C. Benvenuti and R. S. Calder, Le Vide Suppl. 157, 29 (1972). 